the factors causing stress; the requirements for particular jobs, how stress and fatigue in them can be reduced and assessed; the selection of the worker for a particular job and his supervision. The chemical environment is evaluated in a contribution by Dr. John A. Zapp, Jr.

The book is of value in that it presents an aspect of industrial work which has in the past received little consideration, while its well-documented factual content will provide more than an interesting introduction to some of the problems of human engineering with which the physician, the manager, and the engineer in industry should be acquainted.

\section{P. Priban}

Occupational First Aid, 2nd ed. (Pp. 73; illustrated. 4s. 6d.) London: The St. John Ambulance Association. 1961 .

The second and improved edition of Occupational First Aid published by the St. John Ambulance Association has now made its appearance.

It is more profusely illustrated although the two-colour illustrations are rather lurid and might be clearer in black and white.

In accordance with current practice the Association has recognized that first aiders in industry do perform treatment for minor injuries and illnesses without medical supervision, and it has devoted almost one-third of the book to this subject.

There are two points of criticism. Both sterility and no-touch dressing techniques are difficult subjects to teach to first aiders, and it appears unwise to the reviewer to show a dressing trolley set up with mixed sterile and non-sterile objects on the same shelf. It is an accepted principle that the upper shelf should contain sterile objects only and that such non-sterile requisites as bandages and the cetrimide bottle should be confined to the lower shelf.

The other point concerns haemorrhage. There is no mention of elevation of the limb and of lying the patient down before proceeding to the final and dangerous step of applying a rubber constrictive bandage. There are many people who believe that this appliance should be banished from the armamentarium of the first aider.

Despite these criticisms this edition will be read widely by first aiders in industry and should also be studied by managers who have the responsibility for providing first aid services in their factories-especially if they are not guided by the advice of a doctor trained in industrial medicine.

\section{R. A. TREVETHICK}

A Survey of Some Current Surgical Dressing Techniques. Report of an Occupational Health Section Scholarship Project (Studies in Nursing No. 2). By M. Margaret Williams. (Pp. 76; 6 figures. 6s. 6d.) London: The Royal College of Nursing. 1961.

The Royal College of Nursing has published a survey of surgical dressing techniques carried out in the medical departments of a cross-section of industry in this country. It is a report which should be read and digested by all doctors and nurses who perform or are responsible for treatment in industry, and it should serve as a yardstick against which their own techniques can be measured.

Some 35 suspected dangerous practices are discussed, which should form the basis for any heart searching and reorganization of technique. All these faults appear to have been transgressed by some of the respondents, for example, Suspected Dangerous Practice number 35, and perhaps one of the most important, is concerned with the segregation of clean and dirty cases. The cubicle system in the treatment room is probably one of the most effective ways of doing this.

After making comment on all the 35 suspected dangerous practices, a graph is shown in which the sepsis rate, calculated from the cases at risk and the number going septic, is plotted against the number of transgressions of ideal technique. It appears that a higher rate of sepsis exists in those medical departments that commit the greatest number of suspected dangerous practices, and also, as an interesting sideline, it is brought out that heavy industry is more liable to have a high sepsis rate than light industry.

The reviewer would take issue on one point which concerns the definition of sepsis, "the slightest inflammation indicates some degree of sepsis". If this were taken as the criterion for sepsis then almost $100 \%$ of lacerations and burns would be classed as septic since they heal by a process of inflammatory repair. Surely sepsis should be defined as a condition where there is heat, redness, swelling, throbbing pain, lymphangitis, and discharge of pus. Any combination of these signs indicates that the normal process of repair has been superseded by added infection.

This criticism apart, the survey is most helpful in pinpointing pitfalls into which it is too easy to slip. One of the most telling phrases used is the very last one in the book, "all practice should be under constant review". It is surprising to find that there are departments where daily dressings still take place. In the survey it is recommended that dressings should be undisturbed for 24 hours in the first place and then should be looked at every three days. It is even more desirable to the reviewer's mind that the dressing should remain undisturbed for a minimum of five days and on occasions 10 days, and this is quite possible in industry if patients are instructed how to wash and avoid getting their dressings wet. Top bandages can always be replaced if dirty.

Much is made of the use of standard packs ready sterilized for finger and other dressings. Disposable gallipots of aluminium foil and paper towels are all excellent ways of eliminating cross-infection.

This survey deserves a wide distribution, and if its advice is followed there should be a drastic reduction in cross-infection of casualties treated in industry.

\section{R. A. Trevethick}

Tidy's Massage and Remedial Exercises in Medical and Surgical Conditions, 10th ed. Edited and revised by J. O. Wale. (Pp. viii $+600 ; 192$ figures. 42s.) Bristol: John Wright. 1961. 